\title{
Chiral plasmonic nanostructure of twistedly stacked nanogaps
}

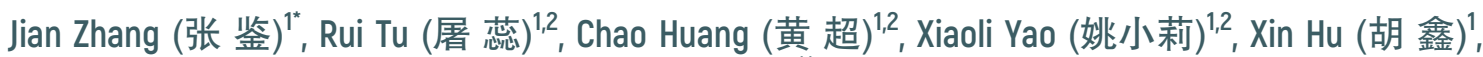 \\ Haixiong Ge (葛海雄) ${ }^{3}$, and Xuefeng Zhang (张雪峰) $)^{\text {t** }}$ \\ ${ }^{1}$ Institute of Advanced Magnetic Materials, College of Materials \& Environmental Engineering, Hangzhou Dianzi University, Hangzhou 310018, China \\ ${ }^{2}$ College of Electronics and Information, Hangzhou Dianzi University, Hangzhou 310018, China \\ ${ }^{3}$ Department of Materials Science and Engineering, College of Engineering and Applied Sciences, Nanjing University, Nanjing 210093, China
}

*Corresponding author: jianzhang@hdu.edu.cn

${ }^{*}$ Corresponding author: zhang@hdu.edu.cn

Received July 13, 2020 | Accepted September 4, 2020 | Posted Online November 30, 2020

\begin{abstract}
Nanogap plasmonic structures with strong coupling between separated components have different responses to orthogonal-polarized light, giving rise to giant optical chirality. Here, we proposed a three-dimensional (3D) nanostructure that consists of two vertically and twistedly aligned nanogaps, showing the hybridized charge distribution within 3D structures. It is discovered that the structure twisted by $60^{\circ}$ exhibits plasmonic coupling behavior with/without gap modes for different circular-polarized plane waves, showing giant chiral response of $60 \%$ at the wavelength of $1550 \mathrm{~nm}$. By controlling the disk radius and the insulator layer, the circular dichroism signal can be further tuned between 1538 and $1626 \mathrm{~nm}$.
\end{abstract}

Keywords: metal-insulator-metal structure; nanogaps; gap mode; twisted stacking; charge distribution; chiral response. DOI: 10.3788/COL202119.013601

\section{Introduction}

Localized surface plasmons (LSPs) ${ }^{[1,2]}$ are collective charge oscillations confined within the interface between the noble metal nanoparticles and the surrounding dielectric medium, and the resonance is sensitively related with the structural geometry, material, and environment ${ }^{[3,4]}$. It has been proved that LSP nanostructures with spatial asymmetry own different coupling behaviors for polarized light of opposite handedness and exhibit artificially designed chiral responses ${ }^{[4-8]}$.

Nanogap structures, such as dimers, bowties, and pairs of semicircles and rods, can confine the electromagnetic field into the narrow gaps or at the sides of particles with different incident polarizations ${ }^{[9,10]}$. Due to this polarization-sensitive resonance, the chiral responsibility of nanogap structures is studied. Until now, planar nanogap structures, including dolmen structures ${ }^{[11]}$, split rings ${ }^{[12]}$, and vortex gaps ${ }^{[13]}$, were developed for giant chiral response.

Recently, metal-insulator-metal (MIM) three-dimensional (3D) nanostructures have attracted extensive concerns in the researches of plasmonics. It has been demonstrated that, by stacking layer by layer, the vertically aligned plasmonic structures can induce complicated charge distribution within 3D structures ${ }^{[14-16]}$. Furthermore, the twistedly stacked MIM structures can lead to the 3D-twisted charge distribution and realize higher-order or hybridized modes for chiral response ${ }^{[17-19]}$.
In this work, we studied the chiral response with 3D-stacked nanogap structures. This chiral MIM nanostructure consists of two vertically and twistedly aligned nanodisks with a nanogap, resulting in hybridized charge distribution between two layers. Excited with right-handed circular-polarized (RCP) and lefthanded circular-polarized (LCP) plane waves, the stacked structure twisted at $60^{\circ}$ exhibits plasmonic coupling behaviors with/ without gap modes, respectively, showing a giant chiral response $(\Delta T)$ of $60 \%$ at the wavelength of $1550 \mathrm{~nm}$. This chiral response can be further tuned by controlling the particle size and the insulator layer of the structure.

\section{Methodology}

To understand the hybridization mechanism, plasmonic modes in nanogap structures are investigated firstly. It has been previously proved that nanogap structures own different LSP coupling performances corresponding to the alignment between the polarization and gap ${ }^{[10,20]}$. When the incident polarization is perpendicular to the gap, the near-field intensity is confined within the gap area, called as gap mode [shown in Fig. 1(a), panel (1)]. When the incident polarization is parallel with the gap, the charge distribution and the near-field intensity are located at the sides of the components, which is similar to LSP coupling behavior of single particles (SPs), named the SP mode here [shown in 


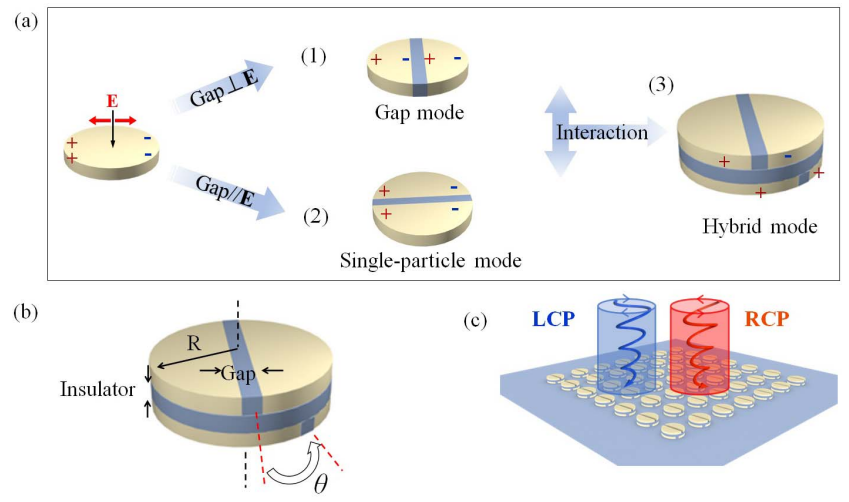

Fig. 1. Schematic diagram of structural model proposed in this study. (a) The formation of the hybrid mode. (b) The twistedly and vertically aligned MIM structure realized with the rotation of $\theta$. (c) The proposed periodic array of the MIM structure with LCP and RCP plane waves.

Fig. 1(a), panel (2)]. By twistedly stacking the two layers, the charge distribution in each gap layer is impacted and distorted by each other, leading to the hybridization of plasmonic resonance [shown in Fig. 1(a), panel (3)].

Figures 1(b) and 1(c) illustrate the proposed periodic MIM nanostructure. The 3D finite-difference time-domain (FDTD) method is carried out using commercial software (FDTD Solution, Lumerical Inc.) to examine the charge distribution, near-field profile, and chiral response. For normal incidence, normalized LCP and RCP plane waves with electric field $E_{0}=$ $1 \mathrm{~V} / \mathrm{m}$ are selected to produce the chiral response, ranging from $900 \mathrm{~nm}$ to $2000 \mathrm{~nm}$. A mesh size of $2 \mathrm{~nm}$ (along the $x, y$, and $z$ directions) is set to ensure the accuracy of electric/magneticfield calculations. To optimize the circular dichroism (CD) signal at the wavelength of $1550 \mathrm{~nm}$, which is one of the most important wavelengths for optical communication, the thickness and radius of the Au nanodisks with the gap size of $50 \mathrm{~nm}$ are set at $50 \mathrm{~nm}$ and $220 \mathrm{~nm}$, respectively. The optical constants of Au are taken from Johnson and Christy $(900-2000 \mathrm{~nm})^{[21]}$. Silica with the refractive index of 1.42 is selected as the substrate. The thickness of the insulator layer is set as $90 \mathrm{~nm}$. The refractive indices for the surrounding medium, insulator layer, and gaps are also set as 1.42 to achieve a uniform environment for LSP coupling. The impact of the refractive index $n$ of the insulator layer is further investigated by varying the value from 1.38 to 1.46. Periodic boundary conditions in the $x$ and $y$ directions are applied with the period $P_{x}, P_{y}=800 \mathrm{~nm}$, and absorbing boundary conditions with a perfect matching layer (PML) are applied along the $\pm z$ directions. The twisted angle $\theta$ between the two layers is varied from $0^{\circ}$ to $90^{\circ}$ with a step of $10^{\circ}$ to investigate chiral response, as illustrated in Fig. 1(b). The distributions of charge and electrical and magnetic near-field are further investigated with varied twisted angles, and the corresponding chiral response is valued by $\mathrm{CD}^{[22]}$ :

$$
\Delta T=T_{\mathrm{RCP}}-T_{\mathrm{LCP}}
$$

\section{Results and Discussion}

The transmission spectra for LCP and RCP plane waves with different twisted angles are investigated firstly. As shown in Figs. 2(a) and 2(b), the LCP and RCP transmission spectra are the same for the twisted angle at $0^{\circ}$ and $90^{\circ}$, since there is no structural asymmetry for these structures. For the twisted angle at $60^{\circ}$, the absorption peak around $1550 \mathrm{~nm}$ [marked in Fig. 2(a)] is discovered in the transmission spectrum for the LCP plane wave, and the transmittance is only $21.3 \%$. In contrast, the transmittance for the RCP plane wave at the same wavelength is $83.5 \%$. The transmission difference proves that there is a giant chiral response for this twisted MIM nanostructure.

Figure 2(c) illustrates the trends (marked as yellow dash lines) of transmission by twisting the structure in details. For the LCP plane wave, the absorption peak is red-shifted originally from $1152 \mathrm{~nm}$ (marked as red star) to $1785 \mathrm{~nm}$ with decreased $\theta$ from $90^{\circ}$ to $0^{\circ}$. Meanwhile, the same absorption for the RCP plane wave is inverted to enhanced transmission with decreased $\theta$. The giant transmission difference with the twisted angle at $60^{\circ}$ at the wavelength of $1550 \mathrm{~nm}$ is noted as green stars in Fig. 2(c). Calculated CD signals $(\Delta T)$ impacted by varied structural parameters are concluded in Fig. S1 of Supplementary Material. Absorption spectra also perform chiral responsivity, shown in Fig. S2 of Supplementary Material.

The transmission difference indicates that the chiral response is impacted by the twisted angle. Correspondingly, charge distributions of the MIM structures twisted from $0^{\circ}$ to $90^{\circ}$ are analyzed at the wavelength of $1550 \mathrm{~nm}$. The results with the twisted angle of $0^{\circ}, 30^{\circ}, 60^{\circ}$, and $90^{\circ}$ are shown in Figs. 3(a)-3(d), respectively. For easy understanding, simplified charge oscillations with the same conditions are illustrated in Figs. 3(e)-3(h). With the twisted angle at $0^{\circ}$, the charge distributions of both
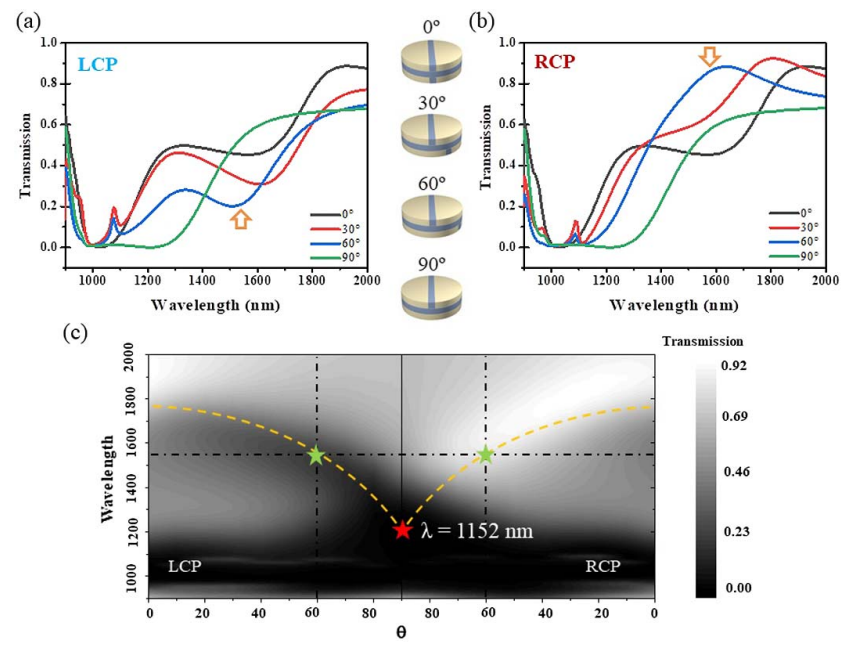

Fig. 2. Transmission spectra of MIM structures for (a) LCP and (b) RCP plane waves with different twisted angles at $0^{\circ}, 30^{\circ}, 60^{\circ}$, and $90^{\circ}$, respectively. (c) The detailed transmission spectra of the MIM structure with twisted angles $\theta$ varied from $90^{\circ}$ to $0^{\circ}$ with a step of $10^{\circ}$ for LCP and RCP plane waves, respectively. The radius of the nanodisks is $220 \mathrm{~nm}$, and the gap in the disk is $50 \mathrm{~nm}$. 


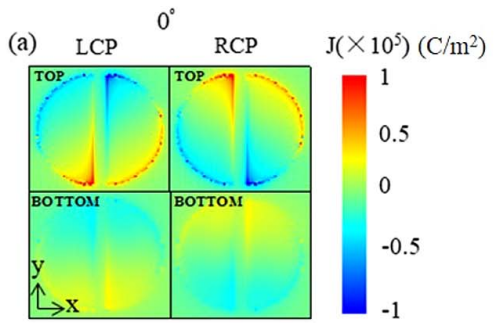

(e)
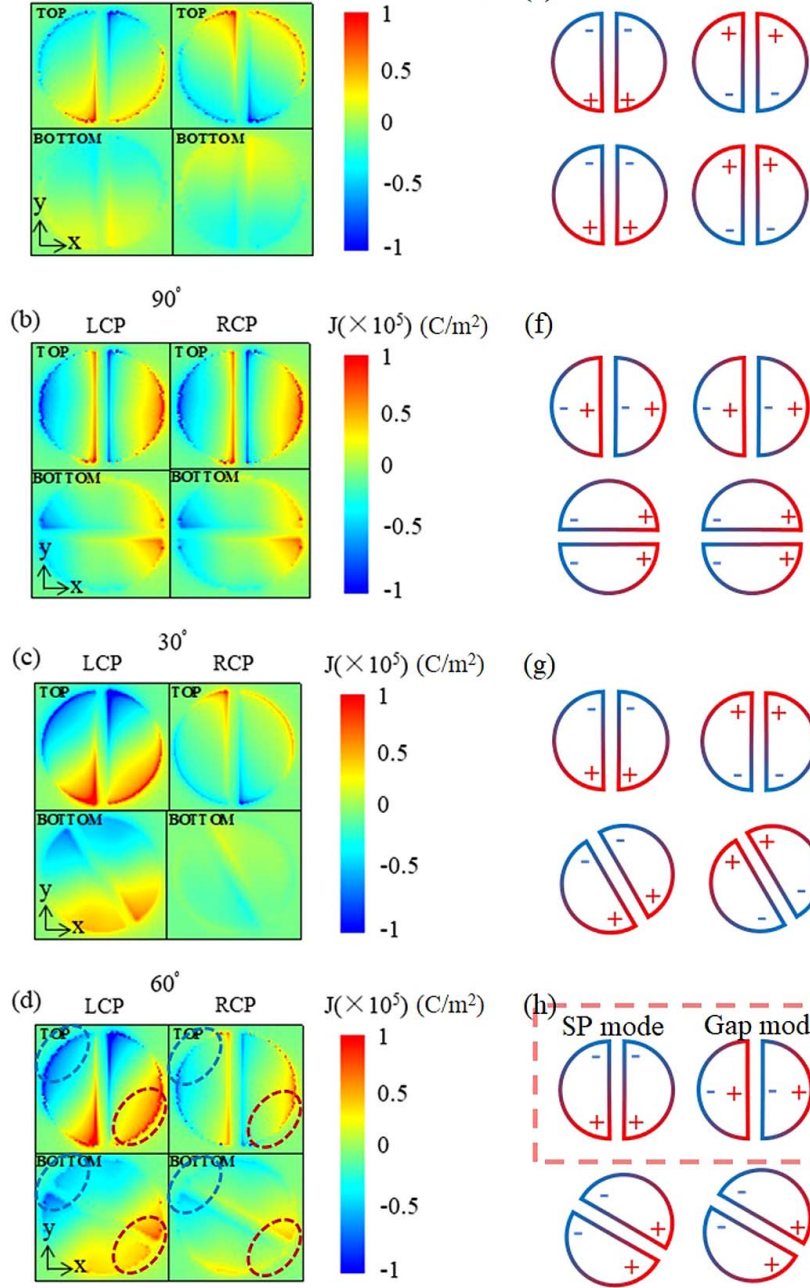

(g)
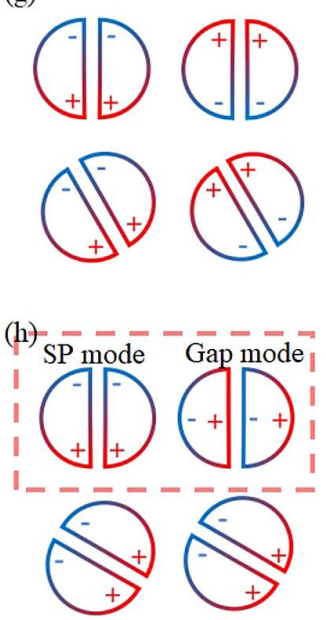

Fig. 3. Simulated charge distributions of the top and bottom nanogaps twistedly stacked with (a) $0^{\circ}$, (b) $90^{\circ}$, (c) $30^{\circ}$, and (d) $60^{\circ}$, excited with LCP and RCP plane waves at the wavelength of $1550 \mathrm{~nm}$, respectively. Simple drawing for charge oscillations of the same structures with the twisted angle of $(e) 0^{\circ}$, (f) $90^{\circ}$, (g) $30^{\circ}$, and (h) $60^{\circ}$, excited with LCP and RCP plane waves at the wavelength of $1550 \mathrm{~nm}$, respectively. Inset in (h): SP mode and gap mode for the same structure with LCP and RCP plane waves, respectively.

LCP and RCP excitations are located at the sides of the particles, showing the typical SP mode [shown in Figs. 3(a) and 3(e)]. With the twisted angle at $90^{\circ}$, the SP mode exists in the bottom layer, and the gap mode exists in the top layer for both LCP and RCP excitations [shown in Figs. 3(b) and 3(f)]. By increasing $\theta$ from $0^{\circ}$ to $30^{\circ}$, the SP mode still exists in the top layer, but the intensity of charge distribution for the RCP condition is weakened, causing the difference of the LSP coupling effect and transmittance [shown in Figs. 3(c) and 3(g)]. Noticeably, for $\theta$ at $60^{\circ}$, the top layer for the LCP plane wave shows the SP mode, while for the RCP condition it diversely shows the gap mode [shown in Fig. 3(d) and marked in the inset of Fig. 3(h)]. For the LCP condition, the charge distribution of the two layers is close to an antibonding-type coupling between two layers [marked in Fig. 3(d)], which is previously convinced in MIM structures ${ }^{[23]}$, while the SP mode of the bottom layer for the RCP condition performs ambiguously with lower intensity. Thus, the antibonding-type coupling for the RCP condition is much weaker than that for the LCP condition, leading to lower absorption and higher transmittance. Corresponding to this difference in coupling modes, the maximum transmission difference achieves $60 \%$ for $\theta$ at $60^{\circ}$.

The optical chirality $C$ for monochromatic electromagnetic radiation in free space is expressed as ${ }^{[24]}$

$$
C \equiv \frac{\varepsilon_{0}}{2} \boldsymbol{E} \cdot \nabla \times \boldsymbol{E}+\frac{1}{2 \mu_{0}} \boldsymbol{H} \cdot \nabla \times \boldsymbol{H},
$$

where $\boldsymbol{E}, \boldsymbol{H}, \varepsilon_{0}$, and $\mu_{0}$ are the electric field, magnetic field, permittivity, and permeability, respectively. It has been proved that chiral metamaterials with spatial asymmetry own different near-field coupling behaviors with different polarizations of incident waves and can enhance the macroscopic chiroptical response ${ }^{[25,26]}$. For chiral structures, the interference between electric dipole and magnetic dipole impacted by structures plays a critical role in the chiral response. The mixed electric dipole and magnetic dipole can be expressed as

$$
\begin{aligned}
& \boldsymbol{p}=\alpha \boldsymbol{E}-i G \boldsymbol{H}, \\
& \boldsymbol{m}=\chi \boldsymbol{H}+i G \boldsymbol{E},
\end{aligned}
$$

where $\alpha$ is the electric polarizability, $\chi$ is the magnetic susceptibility, and $G$ is the mixed electric-magnetic polarizability, presenting a pseudo-scalar for chiral structures. $\boldsymbol{E}$ and $\boldsymbol{H}$ are the electric/magnetic local fields at the structures ${ }^{[27,28]}$. Thus, the coupling between the spin angular momentum of the incident light and the structural chirality induces different electric/ magnetic near-field distributions and impacts the interference between electric dipole and magnetic dipole, leading to the chirality-dependent optical response. Figures 4(a)-4(d) show $|\boldsymbol{H}|$ near-field distributions of the structures twisted with $0^{\circ}$, $30^{\circ}, 60^{\circ}$, and $90^{\circ}$ for LCP and RCP plane waves at $1550 \mathrm{~nm}$, respectively. For the structures twisted with $0^{\circ}$ [Fig. 4(a)] and $90^{\circ}$ [Fig. 4(b)], $|\boldsymbol{H}|$ near-field distributions are symmetric between LCP and RCP conditions, and the $90^{\circ}$-twisted one owns higher $|\boldsymbol{H}|$ field intensity, which could be due to the greater coupling strength of magnetic resonance. For the structure twisted with $30^{\circ}$ and $60^{\circ}$, the $|\boldsymbol{H}|$ field distributions perform differently for LCP and RCP plane waves due to the increased structural chirality, as shown in Figs. 4(c) and 4(d). The greatest difference for $|\boldsymbol{H}|$ near-field distributions is realized for the twisted angle of $60^{\circ}$, and the corresponding $\Delta T$ is over $60 \%$. Such a coupling difference induced by structural chirality is also discovered in $|\boldsymbol{E}|$ near-field distributions shown in Fig. S3 of Supplementary Material. Correspondingly, Fig. 5(a) proves that the CD signal $(\Delta T)$ is maximum when the nanogaps are twisted at $60^{\circ}$, leading to the giant chiral response at $1550 \mathrm{~nm}$, as marked in Fig. 5(b).

Finally, the tunability of chiral behavior of this structure is investigated. The size of the nanodisk impacts the chiral 

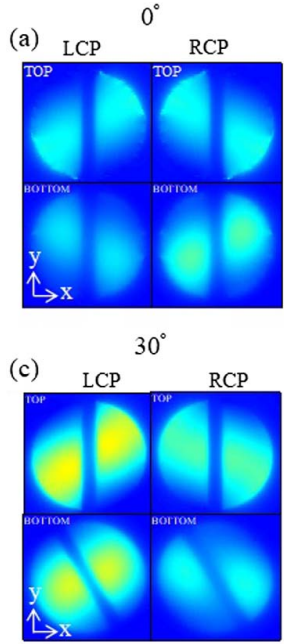

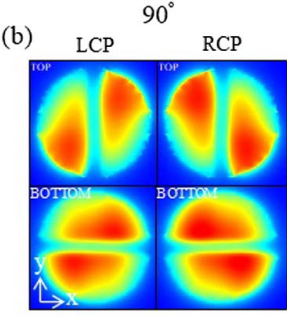

$60^{\circ}$

(d)

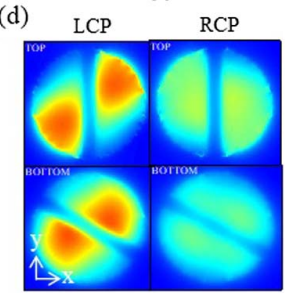

$|H|^{2}\left(\times 10^{-4}\right)\left((\mathrm{A} / \mathrm{m})^{2}\right)$

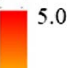

3.4

1.8

0.2
Fig. 4. Calculated magnetic near-field distributions of top and bottom nanogaps twistedly stacked with (a) $0^{\circ}$, (b) $90^{\circ}$, (c) $30^{\circ}$, and (d) $60^{\circ}$, excited with LCP and RCP plane waves at the wavelength of $1550 \mathrm{~nm}$, respectively. (a)

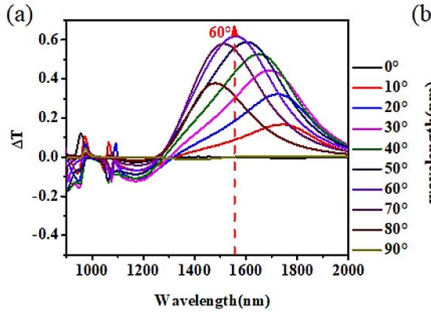

(b)

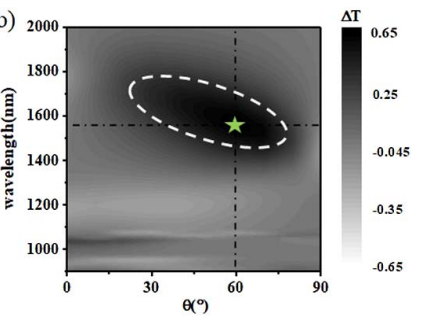

Fig. 5. CD signals of the MIM structure impacted by twisted angles varied from $0^{\circ}$ to $90^{\circ}$. (a) $\Delta T$ spectrum for structures twisted from $0^{\circ}$ to $90^{\circ}$ with a step of $10^{\circ}$. The maximum CD signal is achieved with the twisted angle of $60^{\circ}$, as the red arrow marks. (b) Grayscale image of the CD signal $(\Delta T)$ impacted by the twisted angle and plane wave wavelength. The maximum CD signal is achieved at $1550 \mathrm{~nm}$ for the twisted angle of $60^{\circ}$, as the green star marks. (a)

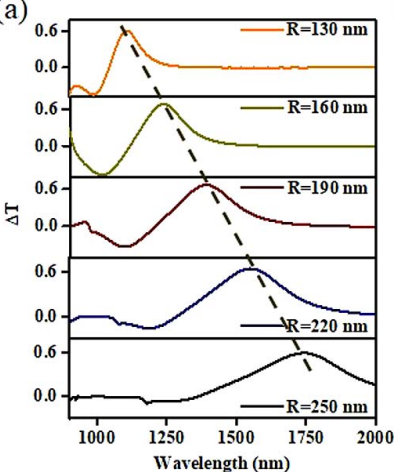

(b)

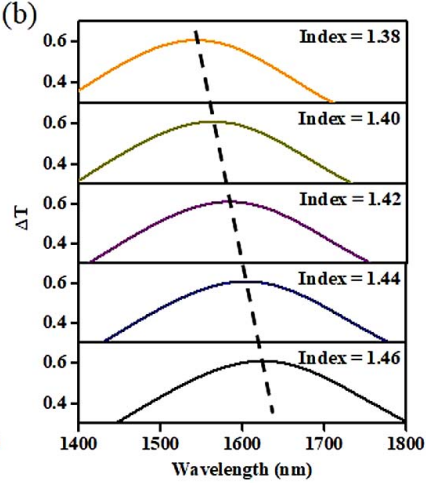

Fig. 6. (a) Maximum of $C D(\Delta T)$ with varied Au nanodisk radius from $120 \mathrm{~nm}$ to $250 \mathrm{~nm}$, in which the red shift with the increasing nanodisk radius is marked by the dash line. (b) Maximum of $C D(\Delta T)$ with varied refractive index $n$ from 1.38 to 1.46 , in which the red shift with the increasing refractive index is marked by the dash line. response, as shown in Fig. 6(a). For the structure twisted at $60^{\circ}$, the maximum $C D$ signal $(\Delta T)$ shows red shift from 1538 to $1626 \mathrm{~nm}$ by increasing the radius of the nanodisk from $130 \mathrm{~nm}$ to $260 \mathrm{~nm}$. The impact of the refractive index $n$ of the insulator layer is also investigated in Fig. 6(b). The chiral response can be tuned from $1538 \mathrm{~nm}$ to $1626 \mathrm{~nm}$ when the $n$ increases from 1.38 to 1.46 , showing critical sensitivity to the change of the medium index.

\section{Conclusions}

We theoretically study the chiral response of twistedly stacked nanogap structures. With the twisted angle of $60^{\circ}$, LSP coupling performance with/without gap modes for RCP and LCP excitation at $1550 \mathrm{~nm}$ is discovered. The electrical and magnetic nearfield distributions of this twisted MIM structure corresponding to different polarizations are further validated. As a result, giant chiral response $(\Delta T=60 \%)$ at the wavelength of $1550 \mathrm{~nm}$ is realized, which shows critical sensitivity to the change of the nanodisk size and the medium index. Our result reveals the hybridization of LSP coupling behavior in twistedly stacked nanogap structures, which perform as a kind of ideal chiral metamaterial.

\section{Acknowledgement}

This work was supported by the National Natural Science Foundation of China (Nos. U1704253 and 51471045), the Zhejiang Provincial Foundation for Distinguished Young Scholars (No. LR18E010001), the Zhejiang Provincial Key Research and Development Program (No. 2019C01121), and the start-up funding supported from the Hangzhou Dianzi University.

\section{References}

1. W. L. Barnes, A. Dereux, and T. W. Ebbesen, "Surface plasmon subwavelength optics," Nature 424, 824 (2003).

2. J. Zhao, X. Zhang, C. R. Yonzon, J. H. Amanda, and P. V. D. Richard, "Localized surface plasmon resonance biosensors," Nanomedicine 1, 219 (2006).

3. K. H. Su, S. Durant, J. M. Steele, Y. Xiong, C. Sun, and X. Zhang, "Raman enhancement factor of a single tunable nanoplasmonic resonator," J. Phys. Chem. B 110, 3964 (2006).

4. X. Huang, C. Lou, H. Zhang, and H. Yang, "Effects of different structural parameters and the medium environment on plasmonic lattice resonance formed by $\mathrm{Ag}$ nanospheres on $\mathrm{SiO}_{2}$ nanopillar arrays," Chin. Opt. Lett. 18, 033601 (2020).

5. Y. Zhao, M.A. Belkin, and A. Alù, "Twisted optical metamaterials for planarized ultrathin broadband circular polarizers," Nat. Commun. 3, 870 (2012).

6. R. C. Devlin, A. Ambrosio, N. A. Rubin, J. P. B. Mueller, and F. Capasso, "Arbitrary spin-to-orbital angular momentum conversion of light," Science 358, 896 (2017).

7. Y. Guo, M. Pu, Z. Zhao, Y. Wang, J. J. Jin, P. Gao, X. Li, X. Ma, and X. Luo, "Merging geometric phase and plasmon retardation phase in continuously shaped metasurfaces for arbitrary orbital angular momentum generation," ACS Photon. 3, 2022 (2016).

8. F. Zhang, M. Pu, X. Li, P. Gao, X. Ma, J. Luo, H. Yu, and X. Luo, "All-dielectric metasurfaces for simultaneous giant circular asymmetric transmission and 
wavefront shaping based on asymmetric photonic spin-orbit interactions," Adv. Funct. Mater. 27, 1704295 (2017).

9. H. Wang, J. Zheng, Y. Fu, C. Wang, X. Huang, Z. Ye, and L. Qian, "Multichannel high extinction ratio polarized beam splitters based on metasurfaces," Chin. Opt. Lett. 17, 052303 (2019).

10. C. E. Talley, J. B. Jackson, C. Oubre, N. K. Grady, C. W. Hollars, S. M. Lane, T. R. Huser, P. Nordlander, and N. J. Halas, "Surface-enhanced Raman scattering from individual Au nanoparticles and nanoparticle dimer substrates," Nano Lett. 5, 1569 (2005).

11. T. Fu, T. Wang, Y. Chen, Y. Wang, Y. Qu, and Z. Zhang, "Chiral near-fields around chiral dolmen nanostructure," J. Phys. D: Appl. Phys. 50, 474004 (2017).

12. A. V. Novitsky, V. M. Galynsky, and S. V. Zhukovsky, "Asymmetric transmission in planar chiral split-ring metamaterials: microscopic Lorentztheory approach," Phys. Rev. B 86, 075138 (2012).

13. C. Kelly, L. K. Khorashad, N. Gadegaard, L. D. Barron, A. O. Govorov, A. S. Karimullah, and M. Kadodwala, "Controlling metamaterial transparency with superchiral fields," ACS Photon. 5, 535 (2018).

14. S. C. Yang, H. Kobori, C. L. He, M. H. Lin, H. Y. Chen, C. Li, M. Kanehara, T. Teranishi, and S. Gwo, "Plasmon hybridization in individual gold nanocrystal dimers: direct observation of bright and dark modes," Nano Lett. 10, 632 (2010).

15. J. Xiao, R. Xiao, R. Zhang, Z. Shen, W. Hu, L. Wang, and Y. Lu, "Tunable terahertz absorber based on transparent and flexible metamaterial," Chin. Opt. Lett. 18, 092403 (2020).

16. M. Hentschel, M. Schäferling, X. Duan, H. Giessen, and N. Liu, "Controlling metamaterial transparency with superchiral fields," Sci. Adv. 3, e1602735 (2017).

17. X. Cui, F. Qin, Q. Ruan, X. Zhuo, and J. Wang, "Circular gold nanodisks with synthetically tunable diameters and thicknesses," Adv. Funct. Mater. 28, 1705516 (2018).
18. N. J. Halas, S. Lal, W. S. Chang, S. Link, and P. Nordlander, "Plasmons in strongly coupled metallic nanostructures," Chem. Rev. 111, 3913 (2011).

19. M. Hentschel, M. Schäferling, T. Weiss, N. Liu, and H. Giessen, "Threedimensional chiral plasmonic oligomers," Nano Lett. 12, 2542 (2012).

20. X. Yao, Z. Shi, C. Li, Z. Kong, G. Zhang, J. Zhang, and X. Zhang, "Tunable optical absorption of dimer nanostructure array achieved by angular evaporation," J. Micromech. Microeng. 28, 115010 (2018).

21. P. B. Johnson and R. W. Christy, "Optical constants of the noble metals," Phys. Rev. B 6, 4370 (1972).

22. Z. Li, W. Liu, H. Cheng, S. Chen, and J. Tian, "Spin-selective transmission and devisable chirality in two-layer metasurfaces," Sci. Rep. 7, 8204 (2017).

23. Y. C. Chang, S. M. Wang, H. C. Chung, C. B. Tseng, and S. H. Chang, "Observation of absorption-dominated bonding dark plasmon mode from metal-insulator-metal nanodisk arrays fabricated by nanospherical-lens lithography," ACS Nano 6, 3390 (2012).

24. K. Y. Bliokh and F. Nori, "Characterizing optical chirality," Phys. Rev. A 83, 021803 (2011)

25. M. Schäferling, X. Yin, N. Engheta, and H. Giessen, "Helical plasmonic nanostructures as prototypical chiral near-field sources," ACS Photon. 1, 530 (2014).

26. A. García-Etxarri and J. A. Dionne, "Surface-enhanced circular dichroism spectroscopy mediated by nonchiral nanoantennas," Phys. Rev. B 87, 235409 (2013).

27. L. Hu, X. Tian, Y. Huang, L. Fang, and Y. Fang, "Quantitatively analyzing the mechanism of giant circular dichroism in extrinsic plasmonic chiral nanostructures by tracking the interplay of electric and magnetic dipoles," Nanoscale 8, 3720 (2016).

28. S. Yoo and Q. H. Park, "Metamaterials and chiral sensing: a review of fundamentals and applications," Nanophotonics 8, 249 (2019). 